\section{Interview with Thomas Powles: The use of durvalumab in urothelial cancer - the latest strides in immuno-oncology}

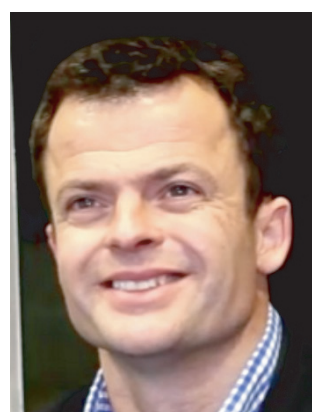

Thomas Powles* speaks to Sebastian Dennis-Beron, Commissioning Editor: Dr. Thomas Powles is a clinical professor of genitourinary oncology and the lead for solid tumor research at Barts Cancer Institute in London. His main research interests areingenital and urinary cancers, in which he leads a spectrum of clinical studies from Phase I to randomized Phase III investigating novel targeted and immune therapies. His research also focuses on correlation of novel biomarkers and aims to define markers with prognostic value that may predict response or resistance to therapy.

Dr. Powles is the author of more than 100 publications, on the editorial board of a number of journals, and part of the faculty for multiple global oncology meetings. He qualified from St Bartholomew's Medical School and received his MD from the University of London.

Accepted for publication: 2 May 2017; Published online: 20 July 2017

\section{Q Could you please provide a brief summary of your career? How did you} become interested in Genitourinary Oncology and in particular Urothelial Cancer (UC)?

I'm a Professor of Urology Cancer at Barts Cancer Center, and I'm the Director of Barts Cancer Center. I did my thesis in mechanisms of resistance to chemotherapy in urology cancer, and that was back in 2000 so I've been interested in this area of research for a long time. In fact, my original research was in this area.

Q How have you seen the field of UC therapy evolve over your time as leader of the genitourinary cancer group at Bart Cancer Institute (London, UK)?

So unfortunately, really nothing much has changed in my lifetime. There were three periods, essentially. There was a period of chemotherapy development that happened in the '70s, ' 80 s and '90s. That reached a plateau probably in the mid-1990s, and then nothing happened for a while. There was then a second period where we attempted to develop targeted therapies, drugs like sunitinib, Herceptin, and vandatinib. That proved to be unsuccessful despite randomized trials. And then there's been this third period of immunotherapy which appears successful and has resulted in positive randomized trials compared with chemotherapy, and therefore immunotherapy may well supersede chemotherapy in the near future in specific areas.

Q What are some of the barriers the field faces when treating UC?

There are three main barriers to treating UC. Number one is we don't know very much about the molecular biology of the tumor. We lag behind other tumor types

*Barts Cancer Center, Barts \& The London School of Medicine \& Dentistry, Queen Mary University of London; thomas.powles@bartsandthelondon.nhs.uk

\section{KEYWORDS}

- clinical trials • combination immunotherapy • durvalumab

- genitourinary cancer

- immunotherapy • PD-L1 inhibitors

- urothelial cancer 
in that regard. We don't have any personalized therapy. Number two is the patients. It tends to occur in the seventh or the eighth decade of life and is linked to smoking cigarettes, so the patients often have comorbidities making them more difficult to treat. And number three is we haven't really had any successful therapies beyond chemotherapy for the last 40 years so the patient's treatment options have been limited.

\section{Q Can you tell our readers about the} Study 1108 and the use of durvalumab in UC patients?

Durvalumab is a PD-L1 inhibitor and it's been tested in a Phase $1 \mathrm{~b}$ study. This study initially enrolled patients who tested positive for the biomarker PD-L1 but then the patient population expanded into all-comer patients. There were two key findings in the 1108 trial - number one is the drug was associated with significant durable response rates in a similar proportion to patients seen with previous immune checkpoint inhibitors in the region of approximately $20 \%[1]$.

The second important finding was there was really quite powerful enrichment into the PD-L1-postive subgroup, suggesting the biomarker may be important in enriching for outcomes $(31 \%$ responses in PD-L1 positives and 5\% in PD-L1 negatives) [1]. This hasn't always been the case with other drugs. It has been for some checkpoint inhibitors, but not all. This biomarker (SP263) and drug pair look particularly promising.

The adverse event profile of durvalumab looks impressive ( $7 \%$ grade $3-4$ adverse events for durvalumab). The common adverse events tend to be rash, pyrexia, and some fatigue. A small proportion of patients get severe toxicity that required intervention [1]. This occurs for all of the immune therapies. One of the key findings is that these drugs appear to be well tolerated when compared with chemotherapy [1].

\section{Q How significant are the survival rates} measured in the trial?

The trial showed progression-free and overall survival. The progression-free survival is short. Median progression-free survival with all immune checkpoint inhibitors in urothelial cancer is short. The drugs are not working by delaying the time to regrowth by parameters established in the chemotherapy era. There are different reasons why that might be the case.
The most powerful reason is I think that a lot of patients had mixed responses, and we define mixed responses as progression of disease.

The second piece, however, is overall survival, and the overall survival of the unselected patients appears to be impressive. We seem to be doing indirect comparisons across other trials, always against historical controls, but we know that historical controls overall survival is in the region of 7 or 8 months. At least in indirect comparisons, it appeared to be numerically longer than that (median $=14$ months for durvalumab). Whether that translates into positive findings in randomized trials remains to be seen.

Q How important is the recent FDA acceptance of review of the Biologics License Application for durvalumab?

The acceptance of review by the FDA of durvalumab is an important milestone because it underpins the clinically meaningful findings from the 1108 trial data. Historically, we have tended to approve drugs based on randomized trials, but in this area of huge unmet need, it seems reasonable to approve drugs where we're showing significant clinical activity, with durable, well-tolerated responses for the first time.

Q Concretely, what does this mean for the use of durvalumab in UC and for patients receiving the treatment?

I think the patients' perspective is that once you've failed chemotherapy, and the majority of patients fail chemotherapy relatively soon after having it, further chemotherapy is of little benefit. Therefore a new approach with a chance for long-term durable outcomes is very attractive for patients. The patient piece is important, and they are driving much of this progress because of the lack of availability of alternative drug therapy beyond immune therapy in this setting.

Q Is there a patient profile that would benefit the most from treatment with the immunotherapy? Are there limitations in using the drug in a wider patient pool?

Personalized therapy with immune checkpoint inhibitors in urothelial cancer is based around two factors. Firstly, the biomarker, and there is a huge amount of uncertainty around the PD-L1 biomarker. It looks like with durvalumab, the biomarker may be important.

The second piece is it looks like patients with liver metastasis don't respond well to therapy. It's 
not clear why that's the case and we will need further randomized trials to define this issue.

\section{Q Why is this tumor particularly responsive} to immunotherapeutics such as durvalumab? Urothelial cancer appears to have a high mutational burden so they are molecularly complex tumors. We know that tumors that are more molecularly complex respond better to immune checkpoint inhibition.

Q Can you tell us about current research into the use of combination immunotherapies in treating genitourinary cancers?

There are three groups of combination therapies in urothelial cancer. The first is the combination of PD-L1 plus CTLA-4, which is durvalumab and tremelimumab, or ipilimumab and nivolumab. Preliminary data with ipilimumab and nivolumab shows response rates in the region of approximately $30 \%$, which appear numerically higher than we've seen with certain monotherapy.

The second group is chemotherapy and immunotherapy, and there are randomized trials testing these in the front-line setting with both pembrolizumab and atezolizumab.

And then the third group is targeted therapy plus immunotherapy, and there are drug trials looking at PARP inhibition, SGS3 inhibition, different targets plus immunotherapy. So these are three separate pathways that are being pursued, and the likelihood is that one or more of these will be exciting and positive.

Q Where do you see the field of UC care in a decade? Will immunotherapies (either as monotherapies or in combination) be at the forefront of treatment?

I think immunotherapies or immunotherapy combinations will supersede chemotherapy.
The question is whether this will be in unselected patients or whether we need to find biomarkers. The drugs will also be moved into the perioperative space, and will be curing more patients with a local disease, a muscle-invasive disease where there is another huge area of unmet need.

\section{Closing Statement}

For patients who failed the previous chemotherapy in urothelial cancer, outcomes were dismal, and quality of life was poor. Immune checkpoint inhibitors like durvalumab are going to offer patients new hope, with long-term durable remissions, better quality of life and improved outcomes.

The story is not complete there - the next chapter is about moving immunotherapy to the front-line space - taking on front-line chemotherapy and potentially beating it either alone or in combination with a CTLA-4 inhibitor, either in all patients or in that important biomarker subgroup. That next step would completely revolutionize the way we treat the disease, and I'm eager to watch this story unfold.

Disclaimer

The opinions expressed in this interview are those of the interviewee and do not necessarily reflect the views of Future Medicine Ltd.

Financial \& competing interests disclosure

The authors have no relevant affiliations or financial involvement with any organization or entity with a financial interest in or financial conflict with the subject matter or materials discussed in the manuscript. This includes employment, consultancies, honoraria, stock ownership or options, expert testimony, grants or patents received or pending, or royalties.

No writing assistance was utilized in the production of this manuscript.

\section{References}

1 Powles T, O’Donnell PH, Massard C et al. Updated efficacy and tolerability of durvalumab in locally advanced or metastatic urothelial carcinoma. (Poster) Presented at: 2017 Genitourinary Cancers Symposium. Orlando, FL, USA. 16-18 February 2017. 